\title{
A CLUMP MODEL FOR WOLF-RAYET STAR
}

\section{ATMOSPHERES}

\author{
A.F. KHOLTYGIN \\ Astronomical Institute, St.Petersburg University, \\ 198904 St.Petersburg, Petrodvoretz, Russia \\ I: afx@aispbu.su
}

\begin{abstract}
There are many indications for the existence of dense cold clumps in WolfRayet (WR) atmospheres, expanding outwards from the stellar core (e.g., Cherepaschuck et al. 1984, St-Louis et al. 1993). Based on this, we consider a clump model for WR model atmospheres. The main features of the model are described. The mechanism of clump structure formation is outlined. The clump model is used for calculating emission line profiles.
\end{abstract}

Key words: stars: Wolf-Rayet - atmospheres - inhomogeneity - line profiles

In clump (or blob) models (Antokhin et al. 1988, 1992; Kholtygin 1988; Cherepaschuk 1990) stellar model atmospheres are proposed consisting of many dense clumps (blobs) embedded in a rarefied interclump medium. The temperature of the proposed clumps is rather low $\left(T_{e} \leq 10^{4} \mathrm{~K}\right)$ in view of their high density (Oskinova et al. 1994).

As was recently shown by Lépine (1993) and Moffat et al. (1993), WR atmospheres probably have an inhomogeneous structure. They identified about $10^{3}$ individual subpeaks in the profiles of the C III $5696 \AA$ line in spectra of WR135 (WC8) and related those to clumps (or eddies) in the atmosphere. Based on the papers cited above we have estimated the parameters of the clumps (lifetime $\tau$, linear size $l$ and He number density $\bar{n}$ vs. velocity dispersion of the subpeaks) in the atmosphere of WR135, which are presented in the Table below.

\begin{tabular}{llrll}
\hline$\sigma_{v}\left(\mathrm{~km} \mathrm{~s}^{-1}\right)$ & $\tau(\mathrm{hr})$ & $l(\mathrm{~cm})$ & $l / R_{*}$ & $\bar{n}\left(\mathrm{~cm}^{-3}\right)$ \\
\hline $10^{*}$ & $\approx 0.2$ & $7.2 \cdot 10^{8}$ & 0.002 & $1.0 \cdot 10^{16}$ \\
$20^{*}$ & $\approx 0.6$ & $4.3 \cdot 10^{9}$ & 0.01 & $1.6 \cdot 10^{15}$ \\
40 & $\approx 2$ & $2.6 \cdot 10^{10}$ & 0.09 & $2.7 \cdot 10^{14}$ \\
60 & $\approx 4$ & $8.6 \cdot 10^{10}$ & 0.2 & $8.1 \cdot 10^{13}$ \\
100 & $\approx 8$ & $2.8 \cdot 10^{11}$ & 0.8 & $2.5 \cdot 10^{13}$ \\
160 & $\approx 17$ & $9.4 \cdot 10^{11}$ & 2.6 & $7.4 \cdot 10^{12}$ \\
\hline
\end{tabular}

Notes: (i) parameters of small clumps expected to be found when the $\mathrm{S} / \mathrm{N}$ ratio has been enhanced; (ii) $R_{*}=5 R_{\odot}$ : radius of the stellar core

Our calculations show that the number density of $\mathrm{He}^{+}$in the clumps becomes large enough to explain the presence of the He I lines in the spectra 
of the WR stars with high effective temperature $\left(T_{\text {eff }} \approx 9 \cdot 10^{4} \mathrm{~K}\right)$ if $p>$ $10^{2}$, where $p$ is the ratio of the density in clump and interclump medium. A mechanism capable of guaranteeing the high clump/interclump number density ratio is compression by shock waves.

We propose the following scenario of clump formation. The primordial density fluctuations in the WR atmospheres appear to be the result of radiation driven stellar wind instability (e.g., Owocki et al. 1990) or thermal instability. The existence of a difference between clump and interclump gas velocities leads to the formation of shock-wave compressed clumps. The complex system of shock waves formed due to numerous perturbations of the velocity field in the atmosphere, is probably responsible for the formation of the inhomogeneous density distribution in WR atmospheres, as revealed by Lépine (1993) and Moffat et al. 1993).

We calculate line-profiles for ions with the low ionization potential (He I $\mathrm{C}_{\text {II }} \mathrm{N}$ II etc.) in a clumped model, taking into account both emission of the clouds and absorption of the stellar radiation by the clouds in front of the core. In a spherically-symmetric model it is hard to explain the existence of the profiles of the He I $\lambda 10830$ lines with bumps of constant shape and velocity displacement in the spectra of some WR stars (Eenens 1991). Therefore, we postulate one or two jets or streams consisting of both rarefied gas and clumps. We have calculated the spectra of these stars in the context of this assumption and have obtained the parameters of the jets.

\section{Acknowledgements}

We are grateful to Dr. I.I. Antokhin for helpful discussions, and Dr. P.R.J. Eenens for providing a copy of his Ph.D. Thesis. We are also thankful to Drs. A.F.J. Moffat and S. Lépine for sending copies of papers before publication. This work was partly supported by grant $\Gamma$ 01.-3.13 of the Russian Ministry of Education and Science.

\section{References}

Antokhin, I.I., Kholtygin, A.F., Cherepaschuck, A.M. 1988, Sov. Astron. 32, 285

Antokhin,I.I., Nugis, T., Cherepaschuck, A.M. 1992, Astron. Zh. 69, 516

Eenens, P.R.J. 1991, Ph.D. Thesis, University of Edinburgh

Cherepaschuck, A.M. 1990, Astron. Zh. 67, 955

Cherepaschuck, A.M., Eaton, J.E., Khaliullin, Kh.F. 1984, ApJ 281, 774

Kholtygin, A.F. 1988, in: Wolf-Rayet stars and related objects (Tallinn), p. 115

Lépine, S. 1994 in press

Moffat, A.F.J. 1994, Ap Space Sci. in press

Oskinova, L.M., Kholtygin, A.F., Feklistova, T.Kh. 1994, Baltic Astronomy submitted

Owocki, S.P., Castor, J.I., Rybicki, G.B. 1988, ApJ 335, 914

St-Louis, N., Howarth, I.D., Willis, A.J., Stickland, D.J., Smith, L.J., Conti, P.S., Garmany, C.D. 1993, $A \& A$ 267, 447 